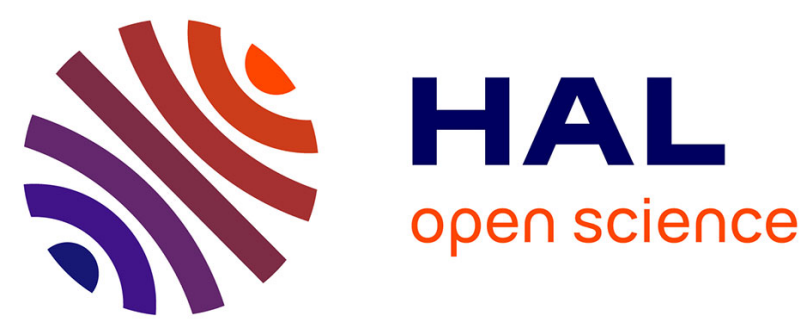

\title{
VISCOSITY OF CONCENTRATED SUSPENSIONS: INFLUENCE OF CLUSTER FORMATION
}

\author{
Victor Starov, V. Zhdanov, Martine Meireles, Catherine Molle
}

\section{To cite this version:}

Victor Starov, V. Zhdanov, Martine Meireles, Catherine Molle. VISCOSITY OF CONCENTRATED SUSPENSIONS: INFLUENCE OF CLUSTER FORMATION. Advances in Colloid and Interface Science, 2002, 96 (1-3), pp.279-293. hal-00323082

\section{HAL Id: hal-00323082 https://hal.science/hal-00323082}

Submitted on 19 Sep 2008

HAL is a multi-disciplinary open access archive for the deposit and dissemination of scientific research documents, whether they are published or not. The documents may come from teaching and research institutions in France or abroad, or from public or private research centers.
L'archive ouverte pluridisciplinaire HAL, est destinée au dépôt et à la diffusion de documents scientifiques de niveau recherche, publiés ou non, émanant des établissements d'enseignement et de recherche français ou étrangers, des laboratoires publics ou privés. 


\title{
VISCOSITY OF CONCENTRATED SUSPENSIONS: INFLUENCE OF CLUSTER FORMATION
}

\author{
V.Starov $^{1 f}$, V.Zhdanov ${ }^{1}$, M.Meireles ${ }^{2}$ C.Molle $^{2}$ \\ ${ }^{1}$ Department of Chemical Engineering, Loughborough University, Loughborough, \\ Leicestershire, LE11 3TU, UK, V.M.Starov@lboro.ac.uk \\ ${ }^{2}$ Laboratoire de Génie Chimique, Université P.Sabatier, Toulouse, France, \\ meireles@lgc.ups-tlse.fr
}

\begin{abstract}
Dispersed particles can form clusters even at sufficiently low concentrations. Colloidal and hydrodynamic forces are responsible for this phenomenon and these forces determine both structure and size of clusters. We assume that viscosity of concentrated suspension is completely determined by cluster size distribution, no matter if clusters form under the action of colloidal, hydrodynamic interactions or shear rates. Based on this assumption an equation, which describes dependency of viscosity on a concentration of dispersed particles taking into account cluster formation, is deduced. Under special restrictions the deduced dependency coincides with the well-known Douherty-Krieger's equation except for a clear physical meaning of entering parameters. Our consideration shows that Douherty-Krieger's equation has deeper physical background than it has been supposed earlier. Experimental verification of the suggested model shows a good agreement with the theory predictions and proves a presence of clusters even at low concentrations of dispersed particles.
\end{abstract}

Key words: cluster size distribution, viscosity, concentrated suspensions.

\footnotetext{
${ }^{f}$ To whom correspondence should be addressed
} 


\section{INTRODUCTION}

There is no need to describe an importance of concentrated suspensions for industrial applications as well as a number of theoretical approaches used for description of viscosity dependence on concentration of dispersed particles [1]. Colloidal and hydrodynamic interactions between particles result in a formation of doublets, triplets and higher clusters [1]. Aggregation of particles is accompanied by destruction of aggregates caused by finite depth of potential well and/or shear stress applied. As a result a distribution of cluster sizes is formed in the suspension. Presence of clusters influences drastically viscosity of concentrated suspensions. Computer simulation is a useful tool for exploring the relationship between interparticle interactions and suspension rheological properties. Clustering and restructuring of colloidal systems under shear is observed in a number of computer simulations [2-6]. However, computer simulations can not substitute analytical modeling and interactions between these two types of modeling can provide a new insight. Here we present a new analytical method, which allows deducing a dependency of viscosity on particle concentration taking into account cluster formation. Differential method, a modified version of which is used in the present paper, has not been frequently used in this area. That is why differential method is briefly reviewed below.

Differential method is adjusted for determination of effective properties of nonhomogenous media. For the first time differential method has been suggested in ref. [7] and then it has been applied for determination of viscosity of concentrated suspensions without cluster formation [8,9]. Elastic properties of solid non-homogeneous materials have been investigated $[10,11]$ with the help of the same method. Below a modified version of differential method is used for calculation of viscosity dependence on concentration in the case when clusters form in a suspension.

\section{THEORY}

Let us consider a suspension of volume $\mathrm{V}$, which contains $\mathrm{N}$ single particles. Volume fraction of particles, $\gamma$, is

$$
\gamma=\frac{v N}{V}
$$

where $v=\frac{4 \pi}{3} a^{3}, \quad a$ are the volume of a single particle and particle radius, respectively. Let $n_{i}, \quad i=1,2,3, \ldots$ be a number of clusters of corresponding size in the volume $\mathrm{V}$ and 
$\gamma_{i, \max }, \quad i=1,2,3, \ldots$ be an averaged packing density of single particles inside clusters. $\mathrm{n}_{1}$ is a number density of “clusters of size 1", that is, single particles, hence, $\gamma_{1, \max }=1$.

Volume fraction of clusters containing i particles can be written as

$$
\gamma_{i}=\frac{V_{i}}{V}, \quad V_{i}=\frac{i v n_{i}}{\gamma_{i, \max }}, \quad i=1,2,3, \ldots
$$

which satisfy the following mass conservation condition

$\sum_{i=1}^{\infty} \gamma_{i} \gamma_{i, \max }=\gamma$

It is assumed below that the viscosity of the suspension is completely determined by cluster size distribution, that is, can be described by the following dependency

$$
\eta\left(\eta_{0}, \gamma\right)=\Psi\left[\eta_{0}, \gamma_{1}, \gamma_{2}, \gamma_{3}, \ldots\right]
$$

where $\eta_{0}$ is the viscosity of the pure liquid.

When all cluster concentrations, $\gamma_{i}$, are zero, the suspension consists only of the pure liquid and Eq (4) gives

$\eta_{0}=\Psi\left(\eta_{0}, 0,0,0, \ldots\right)$,

which is used below.

When particle density, $\gamma$, is small then all $\gamma_{i}$ are also small and Eq (4) can be written in accordance with Einstein's relation as

$$
\Psi\left[\eta_{0}, \gamma_{1}, \gamma_{2}, \gamma_{3}, \ldots\right]=\Psi\left[\eta_{0}, 0,0,0, \ldots\right]+2.5 \sum_{i=1}^{\infty} \eta_{0} \mathbf{A}_{i} \gamma_{i}
$$

where coefficients $A_{i}, \quad i=1,2,3, \ldots$ are deviations of friction coefficient of clusters with $\mathbf{i}$ particles from the corresponding value for solid particles. Obviously $A_{1}=1$. Coefficients $A_{i}, \quad i=1,2,3, \ldots$ are referred to below as friction coefficients for abbreviation. A meaning of friction coefficients can be understood using the following example. Let two spherical particles with the same diameter be considered: the first one is a solid particle, the second one is a particle composed of $\mathbf{i}$ smaller particles (a model of a cluster). A friction force exerted to each of two particles under consideration will be different if both particles are placed in a liquid flow. Let $A_{i}$ be a ratio of the friction force in the case of the composed particle (cluster) to the corresponding force exerted to the solid particle. The friction force is changed in the case of the composed particle because of two reasons: (a) a solid surface exposed to the liquid flow is smaller than in the case of the solid particle, (b) the liquid flow partially 
penetrates inside the composed particle. Two mentioned trends, (a) and (b), change $A_{i}, \quad i=2,3, \ldots$ values in different ways: because of the reason (a) these values decrease and increase because of the reason (b). That is, a complex interplay determines $A_{i}, \quad i=2,3, \ldots$ values. Friction coefficients can be calculated based on theory developed in [12].

Deviation of cluster shapes from spherical is an additional cause of change of friction coefficient values [13].

It is obvious that parameters $\gamma_{i, \max }, A_{i} \quad i=2,3, \ldots$ are functions of both colloidal, hydrodynamic interactions and applied shear rates. These dependencies are left undetermined in the present consideration.

Let a small amount of clusters, $\Delta n_{i}<<n_{i}, \quad i=1,2,3, \ldots$ be marked randomly in the whole volume of suspension. The suspension can be considered as a mixture of the marked clusters surrounded by a suspension of non-marked clusters. Volume concentrations of the marked clusters, $\Delta \mu_{i}, \quad i=1,2, \ldots$, are

$\Delta \mu_{i}=\frac{\Delta V_{i}}{V}, \Delta V_{i}=\frac{i \Delta n_{i}}{\gamma_{i, \max }}, \quad i=1,2,3, \ldots$,

Concentrations of non-marked clusters in the rest of the suspension are

$$
\gamma_{i}-\Delta \xi_{i}=\frac{V_{i}-\Delta V_{i}}{V-\sum_{j=1}^{\infty} \Delta V_{i}}, \quad i=1,2,3, \ldots
$$

or keeping only first order terms:

$$
\Delta \xi_{i}=\frac{V_{i}}{V}-\frac{V_{i}-\Delta V_{i}}{V-\sum_{i=1}^{\infty} \Delta V_{i}}=\frac{\Delta \mu_{i}-\gamma_{i} \sum_{j=1}^{\infty} \Delta \mu_{j}}{1-\sum_{j=1}^{\infty} \Delta \mu_{j}} \approx \Delta \mu_{i}-\gamma_{i} \sum_{j=1}^{\infty} \Delta \mu_{j}, \quad i=1,2,3, \ldots
$$

Marked clusters are surrounded by the rest of the suspension, which is assumed to be a homogenous liquid with viscosity $\Psi\left[\eta^{0}, \gamma_{1}-\Delta \xi_{1}, \gamma_{2}-\Delta \xi_{2}, \gamma_{3}-\Delta \xi_{3}, \ldots\right]$, where small volume fractions $\Delta \xi_{\mathrm{i}}, \mathrm{i}=1,2,3, \ldots$ in the latter expression are given by Eq (8). Hence, viscosity of suspension can be expressed in two ways: according to Eq (4) and considering the suspension as a mixture of marked and non-marked clusters:

$\eta=\Psi\left[\Psi\left[\eta_{0}, \gamma_{1}-\Delta \xi_{1}, \gamma_{2}-\Delta \xi_{2}, \gamma_{3}-\Delta \xi_{3}, \ldots\right], \Delta \mu_{1}, \Delta \mu_{2}, \Delta \mu_{3}, \ldots\right]$

Right hand sides of Eqs (4) and (9) should be equal, this gives

$$
\Psi\left[\eta_{0}, \gamma_{1}, \gamma_{2}, \gamma_{3}, \ldots\right]=\Psi\left[\Psi\left[\eta_{0}, \gamma_{1}-\Delta \xi_{1}, \gamma_{2}-\Delta \xi_{2}, \gamma_{3}-\Delta \xi_{3}, \ldots\right], \Delta \mu_{1}, \Delta \mu_{2}, \Delta \mu_{3 i}, \ldots\right]
$$


A transformation of Eq (10) is given in APPENDIX. The result is the following differential equation for dependence of viscosity of concentrated suspension, $\eta$, on volume concentration of particles $\gamma$

$$
\frac{d \eta}{d \gamma}=2.5 \eta \sum_{i=1}^{\infty}\left[A_{i}+\frac{\sum_{j=1}^{\infty} A_{j} \gamma_{j}}{1-\sum_{j=1}^{\infty} \gamma_{j}}\right] \frac{d \gamma_{i}}{d \gamma}
$$

Solution of Eq (11) should satisfy boundary condition, which follows from Eq (5)

$\eta(0)=\eta_{0}$

Let us introduce the following averaged values

$$
\bar{A}=\frac{\sum_{i=1}^{\infty} A_{i} \gamma_{i}}{\sum_{i=1}^{\infty} \gamma_{i}}, \quad \bar{\gamma}_{\max }=\frac{\sum_{i=1}^{\infty} \gamma_{i, \max } \gamma_{i}}{\sum_{i=1}^{\infty} \gamma_{i}}
$$

which are an averaged resistance coefficient and averaged packing density of all clusters. Using Eq (3) and definition of $\bar{\gamma}_{\max }$ (according to Eq (13)) we can conclude $\bar{\gamma}_{\max }=\frac{\gamma}{\sum_{i=1}^{\infty} \gamma_{i}}, \quad$ or $\sum_{i=1}^{\infty} \gamma_{i}=\frac{\gamma}{\bar{\gamma}_{\max }}$. Substitution of the latter expression and (13) into Eq (11) results in

$$
\frac{d \eta}{d \gamma}=2.5 \eta\left[\frac{d\left(\bar{A} \frac{\gamma}{\bar{\gamma}_{\max }}\right)}{d \gamma}+\frac{\bar{A} \frac{\gamma}{\bar{\gamma}_{\max }}}{1-\frac{\gamma}{\bar{\gamma}_{\max }}} \frac{d\left(\frac{\gamma}{\bar{\gamma}_{\max }}\right)}{d \gamma}\right]
$$

Let us assume that $\bar{A}$ is independent of volume concentration $\gamma$. In this case the latter equation takes the following form

$$
\frac{d \eta}{d \gamma}=2.5 \eta \bar{A}\left[\frac{d\left(\frac{\gamma}{\bar{\gamma}_{\max }}\right)}{d \gamma}+\frac{\frac{\gamma}{\bar{\gamma}_{\max }}}{1-\frac{\gamma}{\bar{\gamma}_{\max }}} \frac{d\left(\frac{\gamma}{\bar{\gamma}_{\max }}\right)}{d \gamma}\right]
$$

or 


$$
\frac{d \eta}{d \gamma}=\frac{2.5 \eta \bar{A}}{1-\frac{\gamma}{\bar{\gamma}_{\max }}} \frac{d\left(\frac{\gamma}{\bar{\gamma}_{\max }}\right)}{d \gamma}
$$

with boundary condition (12).

It is important to emphasise that $\bar{\gamma}_{\max }$ is not supposed to retain a constant value, independent of volume concentration $\gamma$.

Solution of Eq (15) with boundary condition (12) is

$$
\frac{\eta(\gamma)}{\eta_{0}}=\frac{1}{\left(1-\frac{\gamma}{\bar{\gamma}_{\max }}\right)^{2.5 \bar{A}}}
$$

Eq (16) almost coincides with Douherty-Krieger’s equation [14,15]

$$
\frac{\eta(\gamma)}{\eta_{0}}=\frac{1}{\left(1-\frac{\gamma}{\gamma_{\max }}\right)^{[\eta] \gamma_{\max }}}
$$

where $[\eta]$ is an intrinsic viscosity. However, it is important to emphasize that $\gamma_{\max }$ in Douherty-Krieger's equation (16) is constant, whereas $\bar{\gamma}_{\max }$ can not be.

It is easy to conclude that Eqs (16) and (17) coincide if we adopt $\gamma_{\max }=\bar{\gamma}_{\max }, \quad[\eta]=\frac{2.5 \bar{A}}{\bar{\gamma}_{\max }}$.

It is necessary to stress here that in spite of a striking similarity of Eqs (16) and (17) a physical meaning of parameters included in Eq (16) is quite different form those used in Eq (17). According to the theory developed above viscosity dependence on concentration is connected with cluster formation and this physical phenomenon is incorporated in Eq (16).

If particles do not form clusters, that is, $\gamma_{1}=\gamma ; \gamma_{i}=0, i=2,3,4, \ldots$ and, hence, $\bar{\gamma}_{\max }=\bar{A}=1$ should be adopted in Eq (16), which gives

$$
\frac{\eta(\gamma)}{\eta_{0}}=\frac{1}{(1-\gamma)^{2.5}}
$$

The latter equation coincides with earlier obtained solution for the same case $(8,9)$ : suspension of particles, which do not form clusters.

Let us consider in more detail merits and demerits of the suggested method. Merits:

(a) it takes into account clusters formation, 
(b) it takes into account a simplest particle-particle interaction, that is, hard core interaction;

(c) it gives a clear physical meaning of parameters, which are present in Eq(11);

(d) a striking simplicity of this method.

Demerits:

the main demerit of this method is a hypothesis that suspension of non-marked particles can be considered as a homogeneous liquid (mean field approximation). Marked particles (the same as «non-marked ones»!) feel «non-marked particles» as a continuous medium. However, a reasonable agreement of ions' diffusion coefficients calculated according to the Einstein's relation can be considered as a justification of this hypothesis.

\section{COMPARISON WITH KNOWN EXPERIMENTAL DATA}

A comprehensive review of experimental data on viscosity of concentrated suspensions is presented in [16]. In Fig.1 comparison of experimental data with predictions according to Eq (18) (curve 1), and Eq (16) (curves 2-4) is presented. Experimental points used in Fig. 1 are specified in ref. [16]. Fig.1 shows that the whole array of experimental data can be described using Eq (16) at different but reasonable parameters $\bar{\gamma}_{\max }$ and $\bar{A}$ : curve 4 $\left(\bar{\gamma}_{\max }=0.56\right.$, close to a simple cubic packing density; $\bar{A}=0.72$ ); curve 3 ( $\bar{\gamma}_{\max }=0.65$, close to a cubic centered packing density; $\bar{A}=0.67)$, curve $2\left(\bar{\gamma}_{\max }=0.73\right.$, close to hexagonal packing density; $\bar{A}=0.61)$.

\section{EXPERIMENTAL METHOD}

This part presents results of rheological experiments and comparison with theoretical predictions. Yeast suspensions at different cell concentrations are used.

\section{Suspension}

The suspension under investigation was made of active dry baker's yeast Saccharomyces Cerevisiae (Lesaffre, France) suspended in physiological solution (8 g/l $\mathrm{NaCl}$ ). Yeast particles are of $5 \mu \mathrm{m}$ average diameter. The particle density is $1.13 \mathrm{~g} / \mathrm{cm}^{3}$. Volume fraction of the suspension is used below.

\section{Volume fraction determination}

Since yeast cells allow water transfer through the cell's membrane, the determination of the volume fraction is not straightforward. The conversion of a dry weight into the volume fraction has been a source of errors in literature on the concentration dependence of yeast 
viscosity [17]. A particular attention is given here to the determination of the volume fraction, $\gamma$, of yeast suspensions. Reuss et al. [17] proposed a dye dilution technique to determine a volume fraction as a function of a dry weight at several osmotic pressures: a fixed quantity of a dye (Naphtol green B) solution is mixed with the yeast suspension. External water is then determined by measuring the dilution effect.

In this study, the determination of the volume fraction is carried out according to the following procedure: 2-3 drops of concentrated Naphtol green B solution are added to the thoroughly mixed yeast suspension in a $200 \mathrm{ml}$ (Vo) centrifuge tube, which is centrifuged at $5000 \mathrm{rpm}$ for $30 \mathrm{~min}$. The volume of the sediment (Vse) and the extinction value $(710 \mathrm{~nm})$ (E1) of the supernatant are then determined. The sediment is re-suspended in the supernatant of the original suspension in order to ensure constant osmotic pressures. After mixing and centrifuging, volumetric determination of the sediment and measurement of the extinction (E2) are repeated. This procedure gives the following value of the volume fraction

$$
\gamma=\frac{\left(\frac{E 1}{E 2}\right) \cdot V_{S E}-V_{0}}{\left[\left(\frac{E 1}{E 2}\right)-1\right] \cdot V_{0}}
$$

The results obtained are compared with two other techniques of determination of volume fraction: thermogravimetric (Mettler-Toledo) and conductimetric analysis (Coulter). There is a good agreement with data obtained by the three different methods confirming the validity of the experimental results.

The volume fraction $\gamma$ is obtained as a linear function of yeast concentration C (\% dry $\mathrm{w} / \mathrm{w})$ expressed as follows:

$$
\gamma=A * C
$$

The values of A determined by the three different methods are presented in the Table 1.

\begin{tabular}{|c|c|}
\hline Experimental methods & A / (\%dry w/w $)^{-1}$ \\
\hline Dye dilution & 2.1 \\
\hline Conductimetric & 1.8 \\
\hline \multirow[t]{2}{*}{ Thermogravimetic } & 2.0 \\
\hline & $\underline{\text { A average }} \underline{2.0 \pm 0.2}$ \\
\hline
\end{tabular}

Table 1. Experimental values of $A$ using different experimental methods 


\section{Rheological measurements and observation of cluster formation}

Rheological measurements utilized a controlled strain rate rheometer (Rheomat 115 Contraves) with concentric cylinder geometry. Temperature of the suspension in the rheometer was maintained and controlled at $20^{\circ} \mathrm{C}$ in all measurements.

Determination of physical parameters describing the rheological properties of the suspension requires steady state conditions. Experiment shows that at volume fractions higher than 0.3 the rheological behavior becomes non-Newtonian because viscosity value depends on the shear rate.

A linear increasing-decreasing shear rates sequences $10-1000-10 \mathrm{~s}^{-1}$ are performed during 2000s including a step at $1000 \mathrm{~s}^{-1}$ for $200 \mathrm{~s}$ to reach a steady state.

For a direct determination of cluster formation a drop of yeast suspension is placed on the support of the sample and is observed by optical microscope (Zeiss). The number of single particles (N1), clusters (N2), triplets (N3), four and more particle clusters $(\mathrm{N} 4 \&+)$ are calculated.

\section{RESULTS AND DISCUSSION}

The number of single particles (N1), clusters (N2), triplets (N3), four and more particle clusters $(\mathrm{N} 4 \&+)$ at $\gamma=0.002$ volume concentration are presented in Table 2.

Table 2.

\begin{tabular}{|c|c|c|c|c|c|}
\cline { 2 - 6 } \multicolumn{1}{c|}{} & N tot & N 2 & N 3 & N4\&+ & N1 \\
\hline Result & 100 & 6 & 1 & 0 & 85 \\
\hline
\end{tabular}

Yeast suspensions at different low concentration were observed under microscope. Results are presented in Fig. 2 (a, b, c). All used concentrations are low enough ( $\gamma=0.002$, Fig.2a) and ( $\gamma=0.02$ and $\gamma=0.04$ Fig. $2 b$ and $2 c$ respectively). It is usually assumed at theoretical considerations that suspensions are monodisperse at such low concentrations, which is in an obvious contradiction with our observations.

Fig. 3 presents comparison of the measured relative viscosity versus volume fraction of yeast suspension (points) and theoretical Eq (16) (solid line, fitted parameters are $\bar{\gamma}_{\max }=0.73$ and $\bar{A}=1)$. Base fluid is physiological suspension $(8 \mathrm{~g} / \mathrm{l} \mathrm{NaCl})$.

It is worth to remind that according to our derivation parameters $\bar{\gamma}_{\max }$ and $\bar{A}$ are complex functions of (a) interparticle interaction potential, (b) hydrodynamic interactions 
between clusters. Both interactions are unknown in the case of yeast suspensions. In spite of this a reasonable agreement with experimental data are achieved using fitting procedure with these two parameters.

\section{CONCLUSIONS}

A new method is suggested for derivation of viscosity dependence on a concentration of dispersed particles taking into account cluster formation. We assume that viscosity of concentrated suspension is completely determined by cluster size distribution, no matter if clusters form under the action of colloidal, hydrodynamic interactions or shear rates. Based on this assumption an equation, which describes dependency of viscosity on a concentration of dispersed particles taking into account cluster formation, is deduced. Under special restrictions the deduced dependency coincides with the well-known Douherty-Krieger's equation except for a clear physical meaning of entering parameters. Our consideration shows that Douherty-Krieger's equation has deeper physical background than it has been supposed earlier. Observations of yeast suspensions prove a presence of clusters even at low

concentrations of dispersed particles. Viscosity dependency of yeast suspension on concentration is in a good agreement with the deduced theoretical equation and gives reasonable value of parameters.

\section{ACKNOLEDGEMENT}

V.Starov and V.Zhdanov would like to express their gratitude to the Royal Society for support (grant ESEP/JP/JEB/11159). 


\section{APPENDIX}

Everywhere below only first order terms are kept in the consideration. Using Eq (6) the right hand side of Eq (10) can be transformed as

$$
\begin{aligned}
& \Psi\left[\Psi\left[\eta_{0}, \gamma_{1}-\Delta \xi_{1}, \gamma_{2}-\Delta \xi_{2}, \gamma_{3}-\Delta \xi_{3}, \ldots\right], \Delta \mu_{1}, \Delta \mu_{2}, \Delta \mu_{3 i}, \ldots\right]= \\
& =\Psi\left[\Psi\left[\eta_{0}, \gamma_{1}-\Delta \xi_{1}, \gamma_{2}-\Delta \xi_{2}, \gamma_{3}-\Delta \xi_{3}, \ldots\right], 0,0,0, \ldots\right]+ \\
& +2.5 \sum_{i=1}^{\infty} A_{i} \Psi\left[\Psi\left[\eta_{0}, \gamma_{1}-\Delta \xi_{1}, \gamma_{2}-\Delta \xi_{2}, \gamma_{3}-\Delta \xi_{3}, \ldots\right], 0,0,0, \ldots\right] \Delta \mu_{i}= \\
& =\Psi\left[\eta_{0}, \gamma_{1}-\Delta \xi_{1}, \gamma_{2}-\Delta \xi_{2}, \gamma_{3}-\Delta \xi_{3}, \ldots\right]+2.5 \sum_{i=1}^{\infty} A_{i} \Psi\left[\eta_{0}, \gamma_{1}-\Delta \xi_{1}, \gamma_{2}-\Delta \xi_{2}, \gamma_{3}-\Delta \xi_{3}, \ldots .\right] \mu_{i}= \\
& =\Psi\left[\eta_{0}, \gamma_{1}-\Delta \xi_{1}, \gamma_{2}-\Delta \xi_{2}, \gamma_{3}-\Delta \xi_{3}, \ldots\right]+2.5 \sum_{i=1}^{\infty} A_{i} \Psi\left[\eta_{0}, \gamma_{1}, \gamma_{2}, \gamma_{3}, \ldots\right] \Delta \mu_{i}
\end{aligned}
$$

Further transformation of right hand side of Eq (A1) keeping only first order terms gives

$$
\Psi\left[\eta_{0}, \gamma_{1}-\Delta \xi_{1}, \gamma_{2}-\Delta \xi_{2}, \gamma_{3}-\Delta \xi_{3}, \ldots\right]==\Psi\left(\eta_{0}, \gamma_{1}, \gamma_{2}, \gamma_{3}, \ldots\right)-\sum_{i=1}^{\infty} \frac{\partial \Psi}{\partial \gamma_{i}} \Delta \xi_{i}
$$

Using Eqs (A1) and (A2) Eq (10) transforms into

$2.5 \sum_{i=1}^{\infty} A_{i} \Psi\left[\eta_{0}, \gamma_{1}, \gamma_{2}, \gamma_{i}, \ldots.\right] \mu_{i}-\sum_{i=1}^{\infty} \frac{\partial \Psi}{\partial \gamma_{i}} \Delta \xi_{i}=0$

Substitution of expressions for $\Delta \xi_{\mathrm{i}}$ from Eq (8) into the latter equation and collecting all terms proportional to $\Delta \mu_{i}$ gives

$$
\sum_{j=1}^{\infty} \frac{\partial \Psi}{\partial \gamma_{j}} \gamma_{j}-\frac{\partial \Psi}{\partial \gamma_{i}}+2.5 A_{i} \Psi=0, \quad i=1,2,3, \ldots
$$

Let Eq (A3) be multiplied by $\gamma_{j}$ and summarised over $\mathrm{j}=1,2,3 \ldots$ this gives

$$
\sum_{j=1}^{\infty} \frac{\partial \Psi}{\partial \gamma_{j}} \gamma_{j}=\frac{2.5 \sum_{j=1}^{\infty} \eta A_{j} \gamma_{j}}{1-\sum_{j=1}^{\infty} \gamma_{j}}
$$


Substitution of the latter expression for $\sum_{j=1}^{\infty} \frac{\partial \Psi}{\partial \gamma_{j}} \gamma_{j}$ into Eq (A3) gives

$\frac{\partial \Psi}{\partial \gamma_{i}}=2.5 \eta A_{i}+\frac{2.5 \sum_{j=1}^{\infty} \eta A_{j} \gamma_{j}}{1-\sum_{j=1}^{\infty} \gamma_{j}}$

It is obvious that

$\frac{d \eta}{d \gamma}=\sum_{i=1}^{\infty} \frac{\partial \Psi}{\partial \gamma_{i}} \frac{d \gamma_{i}}{d \gamma}$

where $\gamma$ is the volume fracture of particles. After multiplying of Eq (A4) by $\frac{d \gamma_{i}}{d \gamma}$ and summation over $i=1,2,3 \ldots$ Eq (A4) becomes

$\frac{d \eta}{d \gamma}=2.5 \eta \sum_{i=1}^{\infty}\left[2.5 A_{i}+\frac{\sum_{j=1}^{\infty} 2.5 A_{j} \gamma_{j}}{1-\sum_{j=1}^{\infty} \gamma_{j}}\right] \frac{d \gamma_{i}}{d \gamma}$ 


\section{REFERENCES}

1. Russel, W.B., Saville, D.A., Schowalter, W.R. Colloidal dispersions. 1989, London, Cambridge University Press, 525 pp.

2. Bosis J., Brady J.F., J. Chem. Phys., 80, 5141 (1984).

3. Heyes, D.M., Melrose, J.R., J. Non-Newtonian Fluid mech., 46, 1 (1993).

4. Lodge J.F.M., Heyes D.M., Phys. Chem. Chem. Phys., 1, 2119 (1999).

5. Heyes D.M., Branka A.C., Molec. Phys., 96(12), 1757 (1999)

6. Dickinson E, J. Col. Int. Sci., 225, 2 (2000).

7. Bruggemann, D.A., Ann. Phys., 24, 636 (1935).

8. Brinkman, H.C., J. Chem. Phys. 20, 571 (1952).

9. Roscoe R. British J. Appl. Phys. 3, 267 (1952).

10. Cristensen, R.M. J. Mech. And Phys. Solids. 38, 379, (1990).

11. McLaughlin, A. Intern. J. Engin. Sci. 15, 237 (1977).

12. Perepelkim, P.V., Starov, V.M., Filippov, A.N. Colloid J. of the Russian Academy of Sciences (English Translation), 54 (2), 257 (1992).

13. Happel, J., Brenner, H. Low Reynolds Number Hydrodynamics. Martinus Nijhoff Publishers, Prentice-Hall, 1965.

14. Krieger, I.M., Dougherty, T.J. Trans. Soc. Rheology, III, 137 (1952).

15. Krieger, I.M. Adv. Colloid Interface Sci, 3, 111 (1972).

16. Thomas, D.G. J. Colloid Sci. 20, 267 (1965).

17. Reuss, M., Josic, D.,Ppovic, M., Bronn, W.K. European J. Appl. Microbiol. Biotechnol. 8,167, (1979). 


\section{FIGURE LEGENDS}

Fig. 1 Relative viscosity as a function of volume concentration of dispersed particles.

Experimental points from review [16], solid lines according to Eq(16) with different values of $\bar{\gamma}_{\max }$ and $\bar{A}$

curve $1 \quad \bar{\gamma}_{\max }=1, \bar{A}=1$ (particles do not form clusters)

curve $2 \bar{\gamma}_{\max }=0.73$ (close to hexagonal packing of particles inside clusters), $\bar{A}=0.61$

curve $3 \bar{\gamma}_{\max }=0.65$ (close to cubic centered packing of particles inside clusters), $\bar{A}=0.67$

curve $4 \quad \bar{\gamma}_{\max }=0.56$ (close to simple cubic packing of particles inside clusters), $\bar{A}=0.72$

Fig. 2 Photographs of yeast suspensions at different concentrations
a $\quad \gamma=0.002$
b $\quad \gamma=0.02$
c $\quad \gamma=0.04$

Fig. 3 Comparison of measured and predicted relative viscosity on concentration of yeast suspension. Solid curve according to Eq(16). Fitted parameters are $\bar{\gamma}_{\max }=0.73$ and $\bar{A}=1$. 
Fig.1

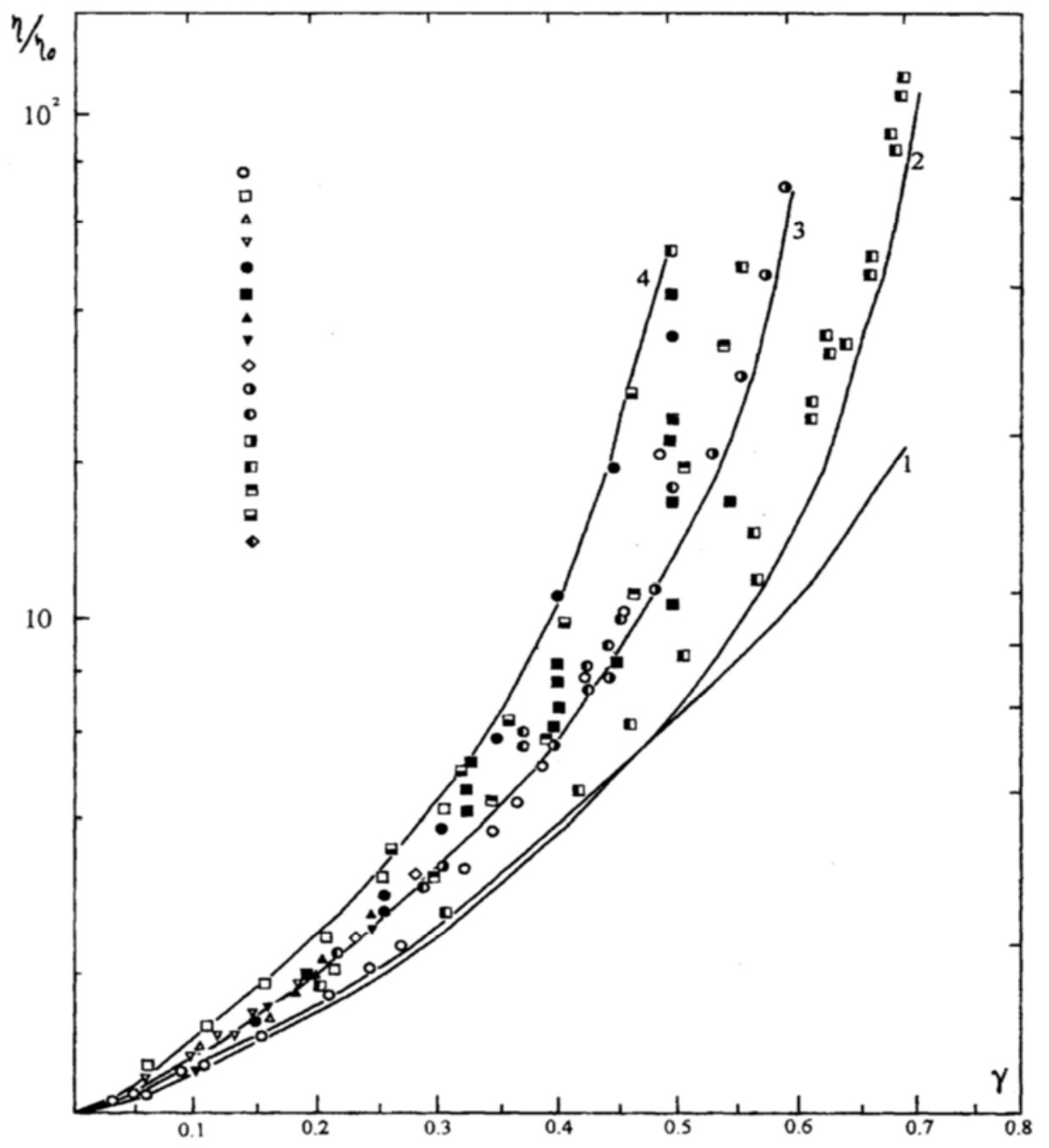




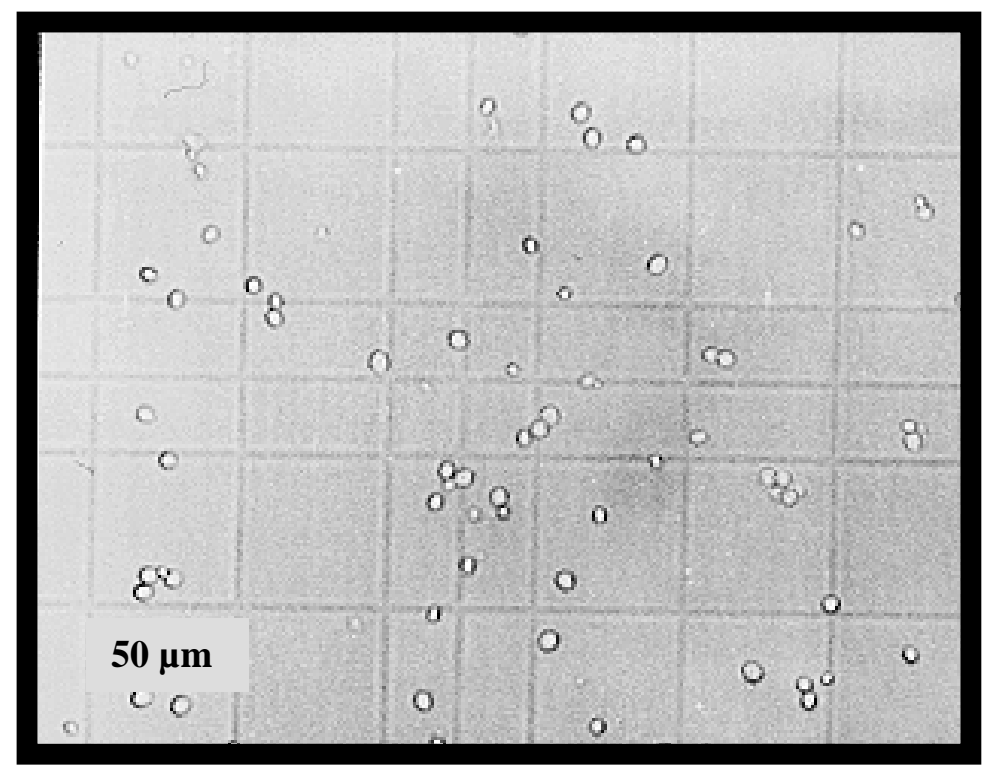

Fig.2a Yeast

suspension, volume

concentration 0.002

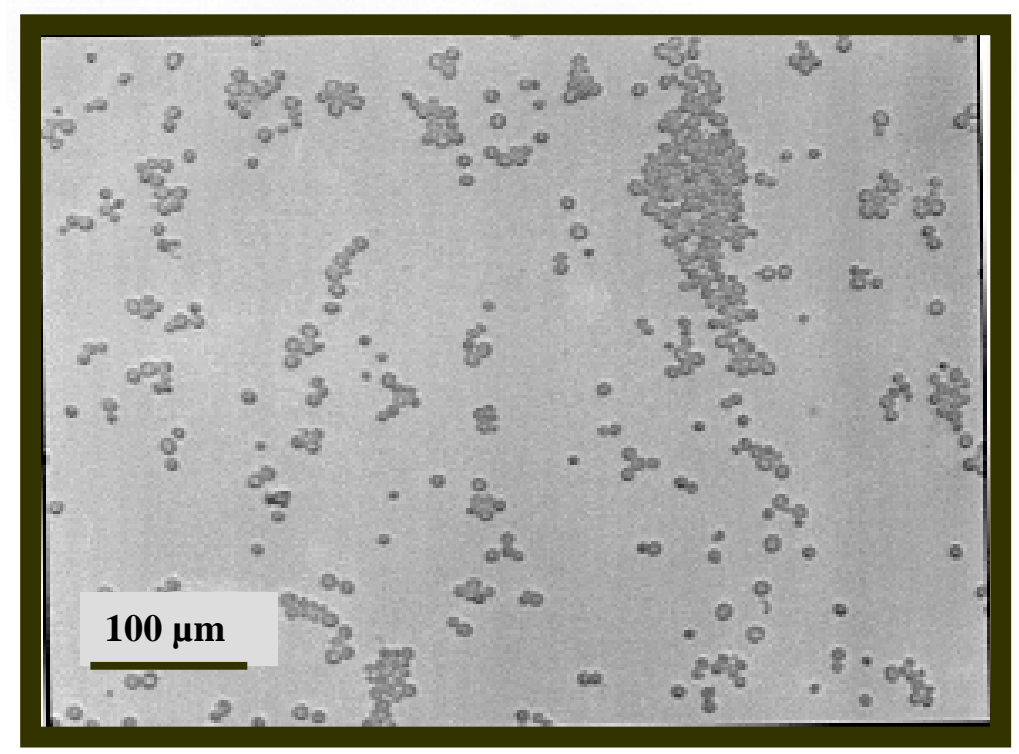

Fig.2b Yeast

suspension, volume concentration 0.02

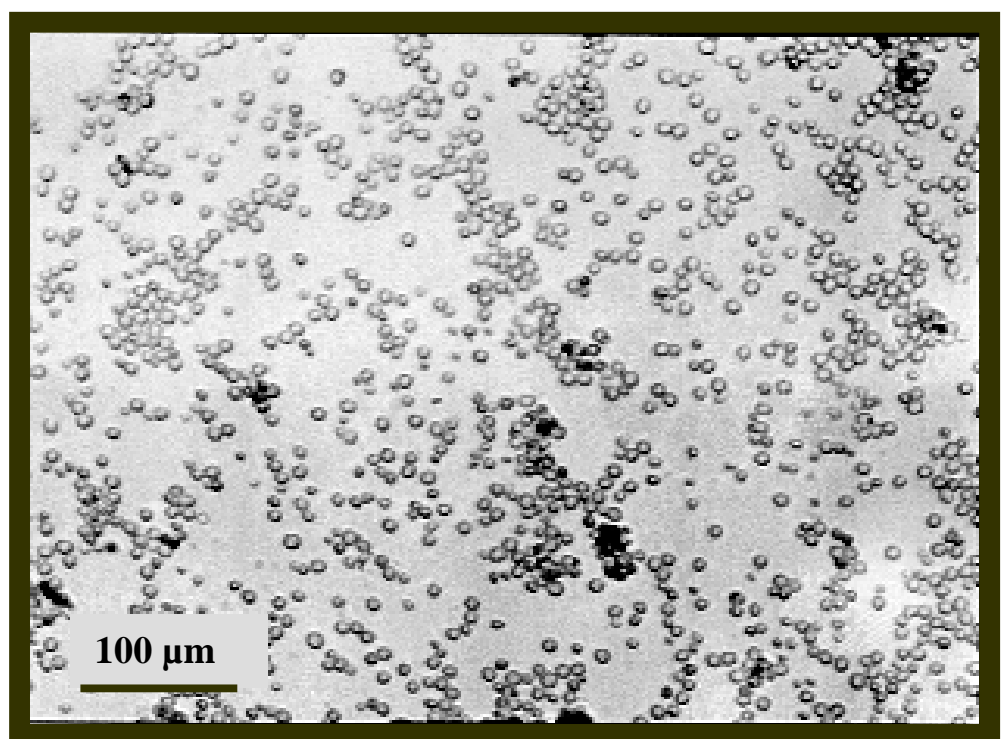

Fig.2c Yeast suspension, volume concentration $\gamma=0.04$ 
Fig. 3

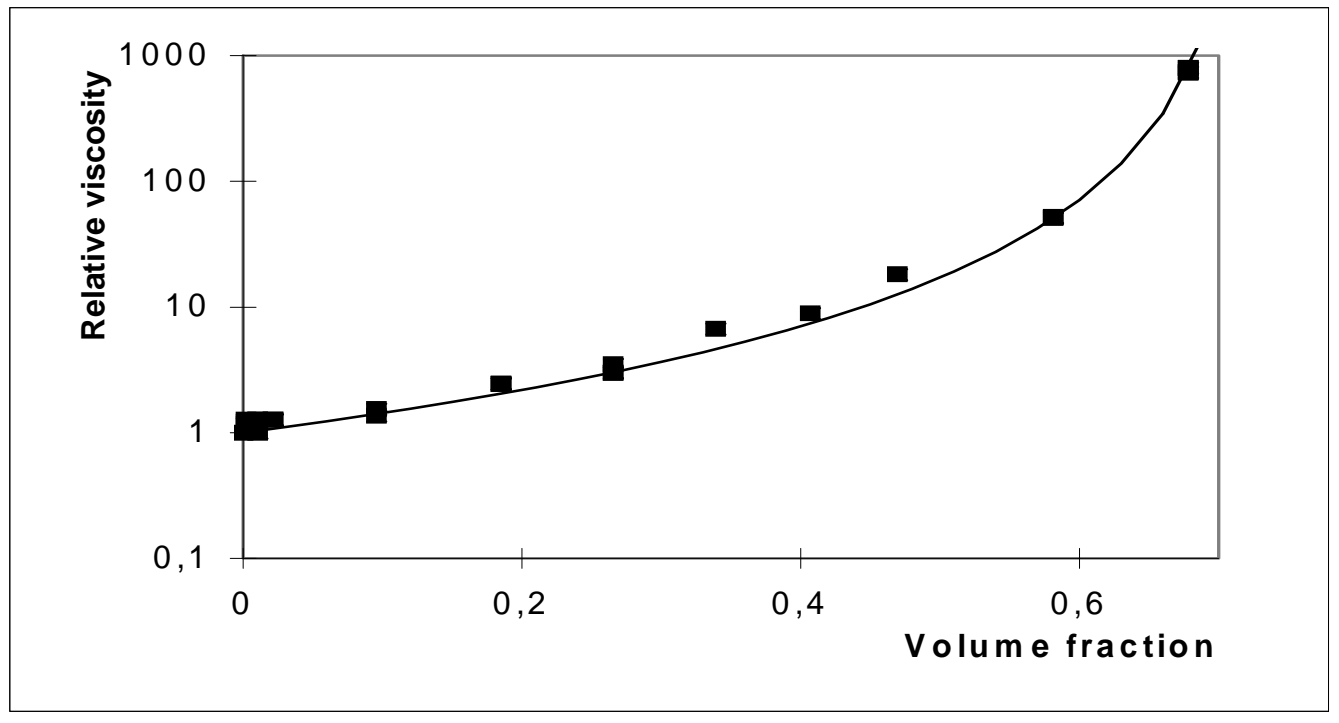

